\title{
Familiäre Bindung als Determinante interkulturellen Erfolgs
}

\author{
Ein Konzept Interkultureller Kompetenz für Expatriates
}

Der Erfolg von Auslandsentsendungen wird in Forschung und Praxis viel diskutiert, der erfolgsfördernde Einfluss familiärer Bindungen bleibt weitgehend unberücksichtigt. In dem Beitrag wird die Rolle der Familie in Entsendungen im Kontext der Bindungstheorie und Interkultureller Kompetenz untersucht und ein Konzept Interkultureller Kompetenz für Expatriates sowie die Umsetzung in der Personalarbeit vorgestellt.

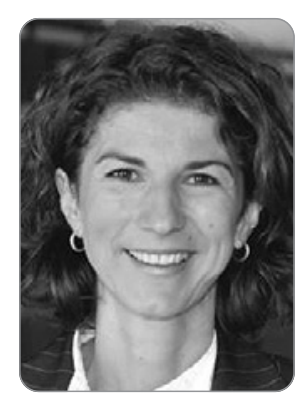

Prof. Dr. Andrea Graf

hat eine Professur für Wirtschaftspsychologie an dem Institut für Recht, Personalmanagement und Personalpsychologie an der Ostfalia Hochschule für angewandte Wissenschaften inne. Bevorzugte Forschungsgebiete: Interkulturelles Management, internationale Personalpsychologie und eignungsdiagnostische Prozessgestaltung.

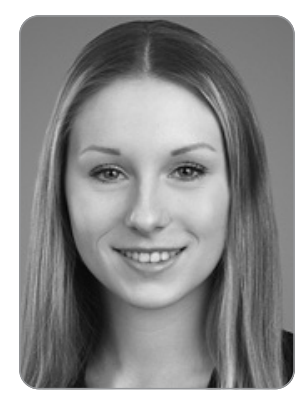

Maren Praetz (LL.B.)

ist wissenschaftliche Mitarbeiterin an dem Institut für Recht, Personalmanagement und Personalpsychologie an der 0stfalia Hochschule für angewandte Wissenschaften. Bevorzugte Forschungsgebiete: Internationales Personalmanagement, internationale Personalpsychologie, Personalrekrutierung.

Stichwörter: Interkulturelle Kompetenz, Auslandsentsendung, familiäre Bindung, emotionale Strukturdimension, interkultureller Erfolg

\section{Problemstellung}

Internationale und interkulturell erfolgreiche Zusammenarbeit ist im Kontext der Globalisierung der Wirtschaft und Märkte sowie einer stetig zunehmenden Internationalisierung der Unternehmen nicht mehr wegzudenken. Folglich sind international einsetzbare Mitarbeiter* mit hoher Inter-

\footnotetext{
* Aus Gründen der besseren Lesbarkeit wird auf geschlechterspezifische Formulierungen verzichtet (z.B. Expatriate, Mitarbeiter). Dennoch sind stets alle Geschlechter gleichermaßen gemeint.
}

kultureller Kompetenz gefragter denn je und die Relevanz von Auslandsaufenthalten und Interkultureller Kompetenz bereits im Studium und im späteren Berufsleben besonders hoch.

Der Erfolg des Auslandseinsatzes ist für alle Beteiligten von wesentlicher Bedeutung. So investiert das Unternehmen immense monetäre Kosten in die Auslandsentsendung und der Mitarbeiter verlässt sein gewohntes Lebensumfeld. Im Rahmen der Vorbereitung der Auslandsentsendung ist die Entscheidung zu treffen, inwiefern der Ehe- oder Lebenspartner und die Kinder in den Prozess der Entsendung integriert werden. Die Personalarbeit wird dabei vor eine Reihe von Herausforderungen gestellt, deren Bewältigung mit den herkömmlichen Methoden und Instrumenten wenig Erfolg versprechend ist. Der Einfluss der Familie auf den Erfolg der Entsendung wird in Theorie und Praxis kontrovers diskutiert. Nachfolgend wird der Stand der Forschung zum Konzept Interkultureller Kompetenz, der Familie als Variable des Auslandserfolges sowie der Bindungstheorie skizziert. Deutlich wird, dass diese drei Themenbereiche das Potenzial signifikanter Wirkungsbeziehungen aufweisen, die bisher noch nicht beleuchtet wurden. Auf dieser Grundlage wird ein Konzept Interkultureller Kompetenz für Expatriates entwickelt und praktische Umsetzungsmaßnahmen für die Personalselektion und -vorbereitung vorgestellt.

\section{Auslandsentsendung und Familie}

\subsection{Stand der Forschung zu Herausforderungen an die entsendete Familie}

In der Literatur werden zahlreiche Problemstellungen und Leistungsanforderungen an den mitreisenden Partner aufgeführt (vgl. Thomas, 2001, S. 70). Die Herausforderungen 
reichen von einem veränderten Zusammenleben mit Partner sowie Kindern über den Umgang mit Einheimischen bis hin $\mathrm{zu}$ organisations- und arbeitsspezifischen Bedingungen. Familienangelegenheiten gelten insgesamt als einer der häufigsten Gründe der Ablehnung einer Auslandsentsendung (vgl. Brookfield Global Relocation Services, 2011, S. 46) und Integrationsprobleme der begleitenden Familie des Expatriates und des Expatriates selbst sind die Hauptgründe für den Abbruch einer Auslandsentsendung (vgl. Deloitte, 2008, S. 27).

Als eine Ursache ist anzunehmen, dass der Auslandseinsatz für den Entsandten und dessen Familie verschiedenartige Folgen hat. Während dieser für den Expatriate in der Regel mit einer Bereicherung der Arbeitsaufgaben, einem höheren Gehalt und einem wesentlichen Karriereschritt einhergeht, ist damit für den Partner sowie eventuell auch für die Kinder des Entsandten oftmals der Verzicht auf den bisherigen Arbeits-, Schul- oder Ausbildungsplatz sowie der Verlust sozialer Kontakte verbunden (vgl. O'Reilly, 2003). Zudem gilt: „Je markanter der Unterschied zwischen der Heimat- und der Gastkultur ist, desto größer ist auch die notwendige Anpassungsleistung, die eine Familie zu erbringen hat" (Grunewald-Petschke, 2015, S. 43).

\subsection{Interkulturelle Kompetenz als Erfolgsfaktor einer Auslandsentsendung}

In der Literatur wird übereinstimmend darauf hingewiesen, dass bei Selektionsentscheidungen für Entsendungen ins Ausland die interkulturellen Fähigkeiten des Bewerbers berücksichtigt werden sollten (vgl. Graf, 2004, S. 3).

In Anlehnung an das Modell Interkultureller Kompetenz nach Graf (2004, S. 60) umfasst dieses die behaviorale, die kognitive sowie die emotionale Dimension. Die behaviorale Strukturdimension Interkultureller Kompetenz beinhaltet verhaltensbezogene Fähigkeiten wie interkulturelle Kommunikationsfähigkeiten. Die kognitive Strukturdimension umfasst, die (Re-)Aktionen des Interaktionspartners und deren Kulturgebundenheit zu erkennen, z.B. Wissen über die Kultur des Interaktionspartners. Unter der emotionalen Komponente Interkultureller Kompetenz wird u.a. die Motivation, sich in eine kulturelle Überschneidungssituation einzubringen, verstanden.

\subsection{Bindungstheorie und Interkulturelle Kompetenz}

Die Bindungstheorie beschäftigt sich damit, dass Menschen ein angeborenes Bedürfnis haben, enge und von intensiven Gefühlen geprägte Beziehungen zu Mitmenschen aufzubauen. Dabei erfüllen sichere Bindungen eine wesentliche protektive Funktion gegenüber auftretenden äußeren Risikofaktoren oder kritischen Lebensereignissen (vgl. Gros- smann, K./Grossmann, K. E., 2012, S. 100). Andererseits wirken sich sichere Bindungen - und dies ist die Besonderheit von Bindungen gegenüber anderen Schutzfaktoren auch ohne das Auftreten von Risikofaktoren positiv auf die weitere Entwicklung eines Menschen aus.

Obwohl die Bindungstheorie zu den am besten fundierten Theorien über die psychische Entwicklung des Menschen gehört und Bindung als zentrales psychisches Grundbedürfnis von Menschen postuliert wird (vgl. Borg-Laufs/Dittrich, 2010, S. 23), wurden diese Erkenntnisse bisher nicht in den Themenbereich der Interkulturellen Kompetenz bzw. Einfluss der Familie bei Auslandsentsendungen übertragen. Dabei drängt sich die Frage auf, inwieweit eine begleitende Familie das Bindungsbedürfnis des Entsandten wesentlich befriedigen kann und damit zur emotionalen Ausgeglichenheit und Stressresistenz beizutragen vermag. Dies wird nachfolgend mit dem Ziel hin untersucht, die geschilderten Forschungsbereiche im Konstrukt der Interkulturellen Kompetenz zu verankern.

\subsection{Stand der Forschung zur begleitenden Familie als Ressource}

Diverse Studien belegen ein stärkendes emotionales Einflusspotenzial von begleitenden Familien. So wurde die protektive Funktion der emotionalen Unterstützung, die von Familienmitgliedern geleistet werden kann, ermittelt (vgl. Caligiuri/Lazarova, 2002, S. 763 ff.). Partnerschaften weisen das Potenzial auf, das Erleben von Belastungen zu reduzieren (vgl. Kappelhoff/Heidemann/Völker/Rietz, 2006, S. 331) und die Lebenszufriedenheit zu erhöhen (vgl. Spieß/Stroppa, 2010, S. 294), wobei die Anpassung des Expatriates an die neuen Bedingungen des Gastlandes signifikant mit der Anpassung des Partners korreliert (vgl. Kittler/Holtbrügge/ Ungar, 2006, S. 134 f.). Die Familie wird von vielen Expatriates als wesentlicher Unterstützungspartner im Umgang mit den Herausforderungen, die eine Auslandsentsendung für sie bereithält, als sehr bedeutend empfunden (vgl. Spieß/Stroppa, 2010, S. 293 ff.). Eine "fehlende" und nicht mitausreisende Familie kann überdies sogar einen Abbruchgrund darstellen (vgl. Grunewald-Petschke, 2015, S. 44).

Insgesamt bleiben Familien länger im Ausland, da der Umzug aufwendiger und kostenintensiver ist und den gegebenenfalls mit ausgereisten Kindern und Jugendlichen Kontinuität geboten werden soll (vgl. Grunewald-Petschke, 2015, S. 44), wobei die verlängerte Aufenthaltsdauer für Unternehmen durchaus von Vorteil sein kann.

Wenig überraschend steht die interkulturelle Anpassung der Expatriate-Familie insgesamt in einem signifikant positiven Zusammenhang zu der Anpassung des Entsandten im Gastland (vgl. Caligiuri/Hyland/Joshi, 1998, S. 607 ff.), so dass die Anpassung der Familie als erfolgskritisch für die 


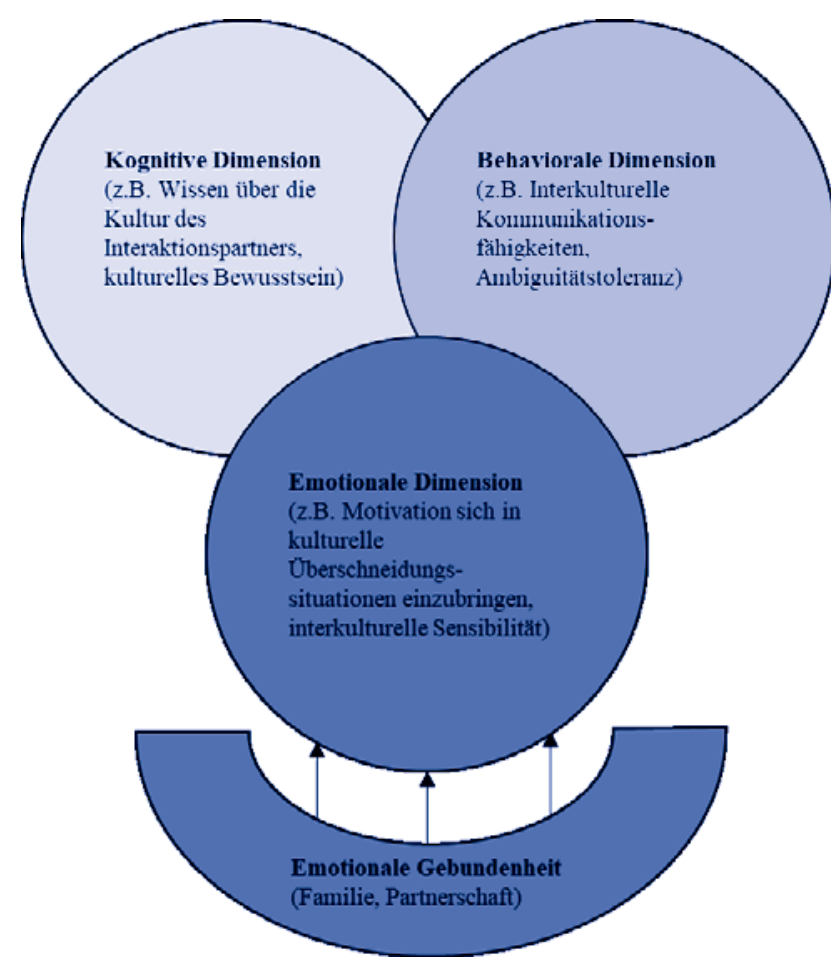

Abb. 1: Konzept Interkultureller Kompetenz für Auslandsdelegierte

Entsendung gesehen werden kann (vgl. Kittler/Holtbrügge/ Ungar, 2006, S. 133 ff.). Ebenso wurde bestätigt, dass die familiäre Situation eines Expatriates bezüglich der Kinder die eigene Adaptation beeinflusst. So ist die Anpassung der Entsandten am höchsten, wenn die Kinder im Gastland geboren wurden und am niedrigsten, wenn sie vor Ende der Entsendung in das Heimatland zurückgekehrt sind.

Wie bereits im Zusammenhang mit den Berichten über die häufig familiär begründeten Abbruchquoten bei Entsendungen beschrieben, kann die Familie auch einen hinderlichen Einfluss haben. Dezidiert weisen Lazarova/Westman/Shaffer (2010, S. 98 ff.) im Rahmen eines Interaktionsmodells nach, dass die wechselseitige Wirkung zwischen Expatriate und der begleitenden Familie insbesondere in Abhängigkeit der kulturellen Anpassung der Familie und deren rollenspezifischer Adaptation sowohl positiv als auch negativ geprägt sein kann. Hiermit wird die Bedeutung der Anpassung der Familie erneut bestätigt.

\section{Konzept Interkultureller Kompetenz für Expatriates und Umsetzung in der internationalen Personalarbeit}

Hochqualifizierte Aufgaben einer Entsendung in interkulturellen Überschneidungssituationen, die ausgeprägter Interkultureller Kompetenz bedürfen, sollten nicht mit „alltäglichen“ interkulturellen Interaktionen gleichgesetzt werden. Das Konzept Interkultureller Kompetenz für Auslandsentsendungen sollte mit Bezug auf die vorangegangenen Überlegungen die emotionale Strukturdimension um die emotionale Gebundenheit einen Expatriate begleitende
Familie erweitern. Mit zunehmender Anpassung des Partners sowie der Kinder, deren Zufriedenheit und harmonischem Zusammenleben erhöht sich die Wahrscheinlichkeit des stärkenden, protektiven Einflusses der Familie auf den Entsandten.

Eine entsprechende Konzeption in Anlehnung an $A b b .1$ kann als Grundlage für optimierte Selektions- und Vorbereitungsmethoden in der Praxis dienen.

Die Selektion von Expatriates umfasst auf Grundlage des Konzepts folgende Bereiche:

Zum einen sollten die drei Strukturdimensionen Interkultureller Kompetenz des Bewerbers erfasst werden. In der Praxis beruht die Selektion der Expatriates oftmals immer noch vorrangig auf bisher erbrachten fachlichen Leistungen (vgl. Holtbrügge/Welge, 2015, S. 329 ff.). Da sich die zu besetzende Stelle im Gastland fachlich stark von der im Heimatland unterscheiden kann und die Interkulturelle Kompetenz als Erfolgsfaktor in der Auslandstätigkeit identifiziert werden kann, sollte diese dezidiert erfasst werden. Hierzu eignen sich z.B.

- Erfahrungswerte über die interkulturellen Fähigkeiten des Bewerbers in vergangenen internationalen Projekten,

- Durchführung von Interkulturellen Assessment Centern bei der die jeweiligen Aufgaben (Fallstudie, Mitarbeitergespräch, Gruppendiskussion etc.) mit interkulturellen Aufgabenstellungen konzipiert werden und alle drei Strukturdimensionen Interkultureller Kompetenz erfasst werden können.

Zum anderen sollte die emotionale Bindung des Expatriates an die Familie erfasst werden.

Hierzu eignen sich z.B.

- Interviews mit den einzelnen Familienmitgliedern über Interesse und Bereitschaft an und für Auslandsaufenthalt, Klärung beruflicher Möglichkeiten bei Dual-CareerCouples, schulische Anforderungen der Kinder im Ausland

- Gespräche mit der gesamten Familie, ggf. unter Beteiligung von Psychologen für eine Einschätzung über (problematische) Strukturen in der Familie.

Für die Vorbereitung auf Auslandsentsendungen weisen die Befunde klar darauf hin, die Familie als Ganzes vorzubereiten und nicht ausschließlich den Expatriate. Hierzu eignen sich z.B.

- Sprachkurse, interkulturelle Trainings oder landeskundliche Informationsveranstaltungen für jedes Familienmitglied zur Stärkung der kognitiven sowie behavioralen Strukturdimension Interkultureller Kompetenz

- Look-and-See Trips für die Familie, um sich einen Eindruck über das Entsendungsland zu verschaffen und hinsichtlich der emotionalen Strukturdimension eine bessere Entscheidungsgrundlage für die Annahme eines Entsendungsangebotes zu erhalten 
- Unterstützung des begleitenden (Ehe-) Partners bei der Suche beruflicher Aufgaben

- Unterstützung der Familie bei der Auswahl geeigneter Schulen der Kinder.

\section{Fazit}

Obgleich die einschlägige Forschung der letzten Jahre vorwiegend die hinderlichen Einflussfaktoren durch eine mitreisende Familie eines Expatriates untersucht und bestätigt, belegt die vorliegende Analyse von Erkenntnissen zum Konstrukt der Interkulturellen Kompetenz, zur Bindungstheorie und theoretischen sowie empirischen Befunde das Erfolgspotenzial von Familien für eine zielführende Entsendung. Die emotionale Gebundenheit in der Familie und deren stärkender Rückhalt kann eine signifikant protektive Wirkung auf die diversen Herausforderungen und Stressoren eines Expatriates sein.

Die im Beitrag entwickelte Konzeption Interkultureller Kompetenz für Auslandsdelegierte erhöht die Anforderungen für internationale Personalabteilungen um ein weiteres Selektions- und Entwicklungskriterium bezüglich der emotionalen Komponente, die neben kultureller Motivation auch die emotionale Gebundenheit der Person umfasst. Geht ungeachtet rechtlicher Vorgaben unter vorgehaltener Hand oft der Single ohne mögliche hemmende und kostenintensive Familie bevorzugt in die Auswahl ein, so ist vielmehr der Kandidat besonders erfolgsversprechend, der während des Auslandsprojektes durch eine intakte, gut vorbereitete Familie (oder Partnerschaft), die dem Auslandsaufenthalt in privater, beruflicher sowie schulischer Hinsicht positiv gegenübersteht, begleitet wird und somit auch in schwierigen Phasen emotional gebunden und damit aufgefangen und gestützt wird. Dies bedarf jedoch die Einbeziehung der einzelnen Familienmitglieder in die Personalselektion sowie in die Vorbereitung auf den Auslandsaufenthalt.

\section{Literatur}

Borg-Laufs, M., K. Dittrich, Die Befriedigung psychischer Grundbedürfnisse als Ziel psychosozialer Arbeit, in: M. Borg-Laufs, K. Dittrich (Hrsg.), Psychische Grundbedürfnisse in Kindheit und Jugend, Tübingen 2010, S. 23-38. Brookfield Global Relocation Services (Hrsg.), Global Relocation Trends 2011 Survey Report, 2011.

Caligiuri, P., M. Hyland, A. Joshi, Testing a Theoretical Model for Examining the Relationship Between Family Adjustment and Expatriates' Work Adjustment, in: Journal of Applied Psychology, Vol. 83 (1998), Nr. 4, S. $598-614$

Caligiuri, P., M. Lazarova, A model for the influence of social interaction and social support on female expatriates' cross-cultural adjustment, in: The International Journal of Human Resource Management, Vol. 13 (2002), Nr. 5, S. 761-772.

Deloitte \& Touche GmbH Wirtschaftsprüfungsgesellschaft (Hrsg.), Entsendungsmanagement im Wandel - Eine Studie über Veränderungen im Entsendungsmanagement und was Unternehmen tun können, um ihre Mitarbeiter erfolgreich entsenden, entwickeln und halten zu können, 2008.

Graf, A., Interkulturelle Kompetenzen im Human Resource Management Eine empirische Analyse zu konzeptionellen Grundfragen und der betrieblichen Relevanz Interkultureller Kompetenzen, Wiesbaden 2004.

Grossmann, K., K. E. Grossmann, Bindungen - Das Gefüge psychischer Sicherheit, 5. Aufl., Stuttgart 2012.

Grunewald-Petschke, C., Kritischer Erfolgsfaktor Familie, in: Personalwirtschaft, 11. Jg. (2015), S. 43-45.

Holtbrügge, D., M. Welge, Internationales Management - Theorien, Funktionen, Fallstudien, 6. Aufl., Stuttgart 2015.

Kappelhoff, V., K. Heidemann, S. Völker, C. Rietz, Partnerschaft und Auslandseinsatz - Beziehungsdimensionen und ihre Bedeutung für die Qualität des Auslandsaufenthaltes bei Expatriates, in: Zeitschrift für Personalforschung, 20. Jg. (2006), S. 318-342.

Kittler, M., D. Holtbrügge, M. Ungar, Ist die kulturelle Anpassung von Entsandten eine Familienangelegenheit? Eine empirische Untersuchung deutscher Fach- und Führungskräfte und deren Familien im Ausland, in: Zeitschrift für Personalforschung, 20. Jg. (2006), S. 121-140.

Lazarova, M., M. Westman, M. Shaffer, Elucidating the positive side of the work-family interface on international assignments - A model of expatriate work and family performance, in: Academy of Management Review, Vol. 35 (2010), Nr. 1, S. 93-117.

Spieß, E., C. Stroppa, Soziale Unterstützung, Stresserleben und Zufriedenheit beim Auslandsaufenthalt, in: Zeitschrift für Personalforschung, 24. Jg. (2010), S. 290-296.

Thomas, A., Going global!...aber verheizen Sie nicht fahrlässig Ihre besten Mitarbeiter, in: Wirtschaftspsychologie, 3. Jg. (2001), S. 68-75. 